\title{
Stabilised reduced-order model of a non-linear eddy current problem by a Gappy-POD approach
}

\author{
MD Rokibul Hasan ${ }^{1}$, Laurent Montier ${ }^{2}$, Thomas Henneron ${ }^{2}$, Ruth V. Sabariego ${ }^{1}$ \\ ${ }^{1}$ Dept. Electrical Engineering (ESAT), EnergyVille, KU Leuven, 3001, Belgium \\ ${ }^{2}$ Univ. Lille, Centrale Lille, Arts et Metiers ParisTech, HEI, EA 2697-L2EP, F-59000 Lille, France
}

\begin{abstract}
In this paper, a stabilized reduced-order model based on the gappy Proper Orthogonal Decomposition (POD) technique is applied to an eddy-current problem with non-linear material property. A classical magnetodynamic finite element formulation based on the magnetic vector potential is used as reference and starting point to build up the reduced models. The computational complexity of the non-linear problem might be reduced by the DEIM-POD technique, but the eddy current effects may make this method unstable. In this work, we propose an approach which is similar to the gappy method to successfully overcome this instability problem, while keeping the computational cost (time and memory) low.
\end{abstract}

Index Terms-Non-linear materials, reduced-order models, proper orthogonal decomposition, discrete empirical interpolation, gappy-POD, eddy currents, finite-element methods.

\section{INTRODUCTION}

$\mathbf{T}$ HE ACCURATE modelling of electromagnetic devices, considering non-linearities and the dynamic complexity of the system, e.g. eddy current effects, movement etc. is still nowadays a challenge in the computation electromagnetic field. The Finite Element Method (FEM) is widely used and versatile for modelling these phenomena. Though it is extremely expensive as the spatial discretisation yields a huge system of equations with prohibitive computational cost. To reduce the computational cost, Proper Orthogonal Decomposition (POD) based reduced order (RO) modelling has been implemented and proved as a feasible and efficient technique mainly in the linear case [1]. In computational electromagnetics, major works on POD based RO modelling can be found in the literature [2], [3], [4]. However, the POD-ROM is not efficient in case of nonlinearities, due to the computational cost of the repetitive evaluation of the non-linear term. The commonly used approaches for the non-linear ROM are linearization [5], [6] and piecewise polynomial approximation [7], [8]. As a matter of fact, these approaches may not be computationally efficient as the nonlinear function cannot be accurately (generally) approximated with low rank piecewise polynomials [10]. To overcome this problem, the Discrete Empirical Interpolation Method (DEIM) combined with POD has been proposed to tackle a non-linear magnetostatic problem in [11]. It consists in interpolating the non-linear terms of the full system by evaluating only some chosen entries. Although the DEIM facilitates the fast computation of the non-linear term based on the chosen DEIM basis vectors and interpolation points, for some non-linear systems a large number of basis vectors is required [12]. Moreover, when including the eddy current effects, the DEIM-POD technique faces an instability issue [13]. An alternative approach to approximate the non-linear system is the Gappy-POD method, developed by Everson and Sirovich [14]. This POD variant

Corresponding author:
rokib.hasan@esat.kuleuven.be).

allows to handle incomplete or so-called "gappy" data sets [15]. In [15], the Gappy POD (G-POD) is applied to reconstruct unsteady flow problems from limited flow measurement data. The data assimilation problem has been reformulated as a gappy data problem in [16] to simulate ocean dynamics in a reduced manner.

To our knowledge there is no study that has implemented the G-POD technique to deal with the DEIM-POD stability issue in electromagnetics. This paper aims at applying the GPOD to model a non-linear magnetodynamic problem. An error indicator $\alpha$ is proposed and analyzed. The G-POD approach is further compared with the POD and DEIM-POD approaches.

\section{Non-Linear Magnetodynamic Model}

Let us consider a bounded domain $\Omega=\Omega_{c} \cup \Omega_{c}^{C} \in \mathbb{R}^{3}$ with boundary $\Gamma$. The conducting and non-conducting part of $\Omega$ are denoted by $\Omega_{c}$ and $\Omega_{c}^{C}$, respectively. The classical (modified) magnetic-vector-potential $(a-)$ magnetodynamic formulation (weak form of Ampère's law) reads: find $a$, such that

$\left(\nu(b) \operatorname{curl} a, \operatorname{curl} a^{\prime}\right)_{\Omega}+\left(\sigma \partial_{t} a, a^{\prime}\right)_{\Omega_{c}}+\left\langle\hat{n} \times h, a^{\prime}\right\rangle_{\Gamma}=\left(j_{s}, a^{\prime}\right)_{\Omega_{s}}$

holds for all test functions $a^{\prime}$ in a suitable function space; $b=\operatorname{curl} a$ is the magnetic flux density (or induction); $j_{s}$ is a prescribed current density associated with stranded inductor and defined in a subdomain $\Omega_{s} ; \hat{n}$ is the outward unit normal vector on $\Gamma ;(\cdot, \cdot)_{\Omega}$ and $\langle\cdot, \cdot\rangle_{\Gamma}$ denote a volume integral in $\Omega$ and a surface integral on $\Gamma$ of the scalar product of their arguments. We consider isotropic materials with a (possibly nonlinear) magnetic constitutive law $h=\nu(b) b$ (magnetic field $h$, reluctivity $\nu(b)$ ) and electric constitutive law $e=\sigma j=-\sigma \partial_{t} a$ (electric field $e$, induced current density $j$, conductivity $\sigma$ ).

The FE discretisation of (1) with $N$ basis functions (generally edge elements for the $3 \mathrm{D}$ and nodal elements for the $2 \mathrm{D}$ case) for $a$ and $a^{\prime}$ (Galerkin approach), leads to the following system of first-order differential equations:

$$
A \partial_{t} x(t)+B(x(t)) x(t)=c(t)
$$




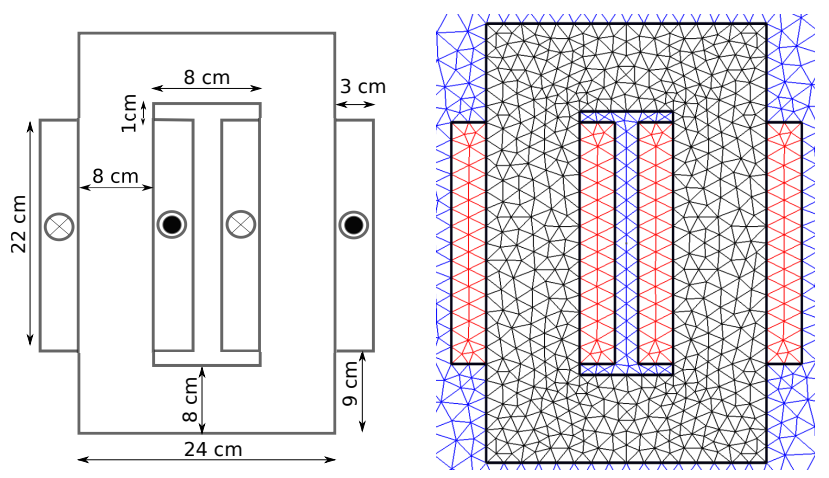

Fig. 1. Geometry (left) and mesh (right) of the single-phase transformer considered for validation.

where $x(t)$ is the time-dependent column vector of $N$ unknowns, $A, B$ are $N \times N$ matrices of coefficients and $c(t)$ is the source column vector (right hand side).

Furthermore, the system (2) is discretized in time by means of the so-called $\theta$-scheme, which amounts to implicit or backward Euler with $\theta=1$, the adopted scheme. A system of algebraic equations is obtained for instant $t_{k}$ with time step $\Delta t=t_{k}-t_{k-1}$.

Taking this into account, discretized system (2) in time reads:

$$
\left[A_{\Delta t}+B\left(x_{k}\right)\right] x_{k}=A_{\Delta t} x_{k-1}+c_{k}
$$

with $A_{\Delta t}=\frac{A}{\Delta t}, x_{k}=x\left(t_{k}\right)$ the solution at instant $t_{k}, x_{k-1}=$ $x\left(t_{k-1}\right)$ the solution at instant $t_{k-1}, c_{k}$ the right hand side at $t_{k}$.

Due to the non-linear material properties, the matrix $B$ depends on the unknown solution vector $x$. Therefore, the system must be solved iteratively. The Newton-Raphson scheme is used to solve the discretized non-linear system $\delta x^{i+1}=$ $\left(J\left(x_{k}^{i}\right)\right)^{-1} r\left(x_{k}^{i}\right)$, where the residual for the $i$-th iteration reads,

$$
r\left(x_{k}^{i}\right)=\left[A_{\Delta t} x_{k-1}+c_{k}\right]-\left[A_{\Delta t}+B\left(x_{k}\right)\right] x_{k}^{i}
$$

with the corresponding Jacobian matrix $J\left(x_{k}^{i}\right)$,

$$
J\left(x_{k}^{i}\right)=A_{\Delta t}+\tilde{J}\left(x_{k}^{i}\right)
$$

$\tilde{J}\left(x_{k}^{i}\right)$ represents the part of the Jacobian that corresponds to the non-linear part of the system (3), i.e. related to $B\left(x_{k}\right)$.

\section{Model ORder Reduction (MOR)}

Model order reduction facilitates the accurate approximation of the large dynamic system behaviour with a reduced cost. In order to project the full system onto a reduced subspace, it is important to generate an appropriate projection operator. Several well known techniques we found in the literature for generating the projection operators. In this work, the projection operators are generated with the POD method. The POD is further combined by DEIM and Gappy procedure, in order to achieve an efficient non-linear reduced model.

\section{A. Proper Orthogonal Decomposition (POD)}

The POD is applied to reduce the FE discretized system (3). In RO techniques, the solution vector $x_{k}$ is approximated by a vector $x_{k}^{r} \in \mathbb{R}^{V \times 1}$ within a reduced subspace spanned by $\Psi \in \mathbb{R}^{N \times V}, V \ll N$,

$$
x_{k} \approx \Psi x_{k}^{r} .
$$

with $\Psi$ an orthonormal projection operator generated from the time-domain full solution $x(t)$ via the snapshots technique [17].

Let us consider the snapshot matrix $S=\left[x_{1}, x_{2}, \ldots, x_{V}\right] \in$ $\mathbb{R}^{N \times V}$ from the set of solutions $x_{k} \in \mathbb{R}^{N \times 1}$ for the selected number of time steps, where $k \in[1 \cdots V]$. Applying the singular value decomposition (SVD) to $S$ as,

$$
S=\mathcal{U} \Sigma \mathcal{V}^{T} .
$$

where $\Sigma \in \mathbb{R}^{N \times V}$ is a diagonal matrix which contains the singular values, ordered as $\lambda_{1}>\lambda_{2}>\ldots>0, \mathcal{U} \in \mathbb{R}^{N \times N}$ and $\mathcal{V} \in \mathbb{R}^{V \times V}$ are left and right orthonormal matrices respectively. We consider $\Psi=\mathcal{U}^{r} \in \mathbb{R}^{N \times M}$, that corresponds to the truncation of $\mathcal{U}$ (i.e. its $M$ first columns, with larger singular values than a prescribed error tolerance $\varepsilon$ ). Therefore, the RO system (3) reads [1]

$$
\left[A_{\Delta t}^{r}+B^{r}\left(x_{k}^{r}\right)\right] x_{k}^{r}=A_{\Delta t}^{r} x_{k-1}^{r}+c_{k}^{r},
$$

where

$$
A_{\Delta t}^{r}=\Psi^{T} A_{\Delta t} \Psi, B^{r}\left(x_{k}^{r}\right)=\Psi^{T} B\left(\Psi x_{k}^{r}\right) \Psi, c_{k}^{r}=\Psi^{T} c_{k}
$$

Note that the rank of $x_{k}^{r} \in \mathbb{R}^{M \times 1}$ in (8) is much smaller than the one of $x_{k}^{r} \in \mathbb{R}^{V \times 1}$ in (6), $M \ll V$ (we should use different notion for $x_{k}^{r}$ but abusing the notation for simplicity).

The RO system (8) can be solved iteratively using Newton Raphson algorithm. Therefore, the residual (4) and the Jacobian equation (5) become

$$
r\left(x_{k}^{r, i}\right)=\left[A_{\Delta t}^{r} x_{k-1}^{r}+c_{k}^{r}\right]-\left[A_{\Delta t}^{r}+B^{r}\left(x_{k}^{r}\right)\right] x_{k}^{r, i}
$$

and

$$
J\left(x_{k}^{r, i}\right)=\underbrace{A_{\Delta t}^{r}}_{M \times M}+\underbrace{\Psi^{T}}_{M \times N} \underbrace{\tilde{J}\left(\Psi x_{k}^{r, i}\right)}_{N \times N} \underbrace{\Psi}_{N \times M} .
$$

The non-linear term in (10) is given by

$$
B^{r}\left(x_{k}^{r}\right)=\underbrace{\Psi^{T}}_{M \times N} \underbrace{B\left(\Psi x_{k}^{r}\right)}_{N \times N} \underbrace{\Psi}_{N \times M} .
$$

We can observe that, the evaluation of $B^{r}\left(x_{k}^{r}\right)$ is still computationally expensive, as the variable $x_{k}^{r}$ needs to be re-projected to the $N$ full dimensional vector space, $\Psi x_{k}^{r}$, and reduce the matrix system (12). Therefore, solving the RO system (8) based on POD method might be as expensive as solving the original system. Furthermore, the computational complexity increases when solving the non-linear system iteratively. As, in each iteration, we need to evaluate not only the non-linear matrix (12), but also the corresponding Jacobian matrix (11) that depends on the original problem size $N$ as well. 


\section{B. Discrete Empirical Interpolation Method (DEIM)}

To overcome the dependency of the RO system to the full system dimension $N$, an efficient method DEIM was proposed in [9], [10]. Let us denote the non-linear function as $f\left(t_{k}\right)=B\left(\Psi x_{k}^{r}\right)$. The DEIM can approximate the $f\left(t_{k}\right)$ on a linear subspace, spanned by the non-linear orthonormal projection operator $W$. This $W$ operator is generated by applying the POD technique to the non-linear snapshot matrix $S_{\mathrm{NL}}=\left[B\left(x_{1}\right) x_{1}, B\left(x_{2}\right) x_{2}, \ldots, B\left(x_{L}\right) x_{L}\right] \in \mathbb{R}^{N \times L}$ similar to (7). We consider $W=\mathcal{U}_{\mathrm{NL}}{ }^{r} \in \mathbb{R}^{N \times D_{e}}$, that corresponds to the truncation of $\mathcal{U}_{\mathrm{NL}}$ (i.e. its $D_{e}$ first columns, which has larger singular values than a pre-defined error tolerance $\varepsilon_{\mathrm{NL}}$ ). The approximation of the non-linear term is given by:

$$
f\left(t_{k}\right) \approx W d\left(t_{k}\right) .
$$

where $d\left(t_{k}\right)$ is the coefficient vector. To obtain the $d\left(t_{k}\right)$, we select appropriate $D_{e}$ rows from the overdetermined system (13). We consider a matrix $P=\left[e_{p_{1}}, \cdots, e_{p_{D_{e}}}\right] \in$ $\mathbb{R}^{N \times D_{e}}$, where $e_{j}$ is the $j$-th column of the identity matrix. The DEIM interpolation indices $p_{1}, \cdots, p_{D_{e}}$ are selected based on the non-linear basis/ projection operator $W$, with a greedy algorithm, see Algorithm 1. In the initial step, the first index $p_{1}$ is selected corresponding to the largest components in magnitude of the first input basis vector $w_{1}$. If $P^{T} W$ is nonsingular, the coefficients $d\left(t_{k}\right)$ can be determined by multiplying (13) with $P^{T}$, leading to the squared system $P^{T} f\left(t_{k}\right)=P^{T} W d\left(t_{k}\right)$,

$$
f\left(t_{k}\right) \approx W d\left(t_{k}\right)=W\left(P^{T} W\right)^{-1} P^{T} f\left(t_{k}\right) .
$$

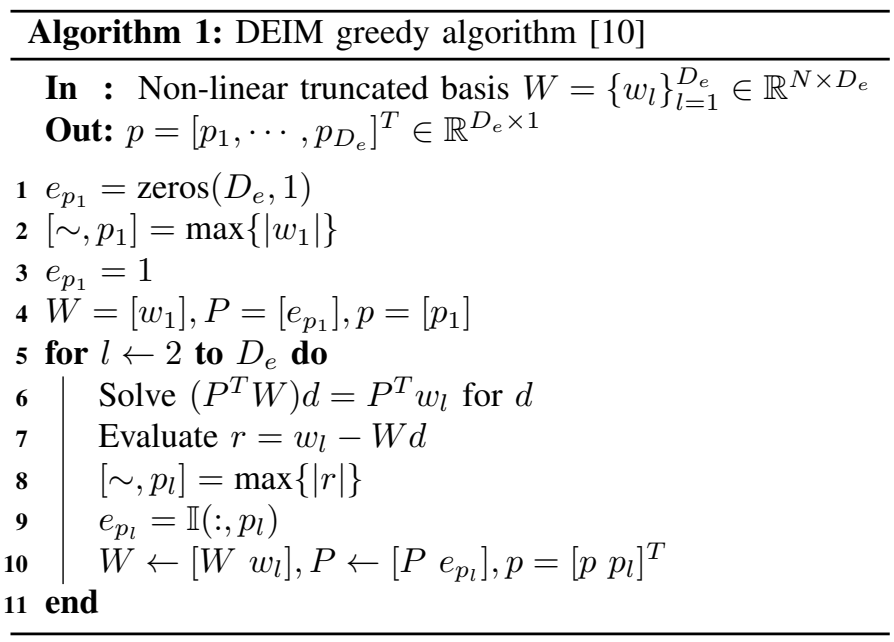

The notation "max" in Algorithm 1 is same as the MATLAB function "max". Therefore, $\left[\sim, p_{l}\right]=\max \{|r|\}$ selects the index $p_{l}$ corresponding to the largest magnitude of the residual $r$.

Inserting the non-linear function $f\left(t_{k}\right)=B\left(\Psi x_{k}^{r}\right)$ in (14), we can get

$$
B\left(\Psi x_{k}^{r}\right) \approx W\left(P^{T} W\right)^{-1} P^{T} B\left(\Psi x_{k}^{r}\right) .
$$

The non-linear matrix $B$ in (15) can be evaluated component wise as $P^{T} B\left(\Psi x_{k}^{r}\right)$, i.e, we need to select only $D_{e}$ components to evaluate the non-linear matrix $B$, which can reduce the computational complexity in a greater extent.

The non-linear term (12) in the reduced form can therefore be written as

$$
B^{r}\left(x_{k}^{r}\right) \approx \underbrace{\Psi^{T} W\left(P^{T} W\right)^{-1}}_{M \times D_{e}} \underbrace{P^{T} B\left(\Psi x_{k}^{r}\right) \Psi}_{D_{e} \times M} .
$$

The approximation of the non-linear term $B^{r}\left(x_{k}^{r}\right)$ does not depend on the full system order $N$ any more. Besides, the matrix $\Psi^{T} W\left(P^{T} W\right)^{-1}$ is time independent and can be precomputed. Similarly, the Jacobian (11) can be approximated as

$$
J\left(x_{k}^{r, i}\right)=\underbrace{A_{\Delta t}^{r}}_{M \times M}+\underbrace{\Psi^{T} W\left(P^{T} W\right)^{-1}}_{M \times D_{e}} \underbrace{P^{T} \tilde{J}\left(\Psi x_{k}^{r, i}\right) \Psi}_{D_{e} \times M} .
$$

\section{Gappy Proper Orthogonal Decomposition (G-POD)}

Although the DEIM-POD can significantlly reduce the computational time to approximate the non-linear $B$ by selecting only $D_{e}$ entries. For some non-linear systems it might require large number $D_{e}$ of the DEIM basis vectors for the accurate representation [12]. However, when including the eddy current effects, the DEIM-POD technique may face instability issues. A stabilization procedure combined with a stability indicator $(\alpha)$ is introduced in this work. The proposed approach consists in increasing the number of selected DEIM components, $\tilde{P}=$ $\left[e_{p_{1}}, \cdots, e_{p_{D_{g}}}\right] \in \mathbb{R}^{N \times D_{g}}$, while keeping the same number of interpolating function $W=\mathcal{U}_{\mathrm{NL}}{ }^{r} \in \mathbb{R}^{N \times D_{e}}$, i.e. $D_{g}>D_{e}$. In this case, this method is similar to the G-POD method. The non-linear term with the G-POD method becomes

$$
B\left(\Psi x_{k}^{r}\right) \approx \underbrace{W\left(\tilde{P}^{T} W\right)^{\dagger}}_{N \times D_{g}} \underbrace{\tilde{P}^{T} B\left(\Psi x_{k}^{r}\right)}_{D_{g} \times N} .
$$

where the $\dagger$ denotes the pseudo-inverse. Note that, if $D_{g}=D_{e}$ enriched then the approach becomes DEIM. An error indicator per instant $t_{k}$ can be introduced from (14) as

$$
\alpha=\tilde{d}\left(t_{k}\right)=\left(\tilde{P}^{T} W\right)^{\dagger} \tilde{P}^{T} B\left(\Psi x_{k}^{r}\right) .
$$

In the DEIM approach the coefficients of $\alpha$ are ordered by decreasing values. These correspond to the guaranteed stable system, but it is not always the case as demonstrated hereafter in practice. We have implemented the G-POD and observed that the $\alpha$ decreases with this method.

\section{2D Single Phase Transformer}

As a test case we consider the single-phase power transformer depicted in Fig. 1. It consists of an iron core and two coils wound around. The coils are made of copper (nonmagnetic, conductivity $\sigma_{C u}=5.7710^{7} \mathrm{~S} / \mathrm{m}$ ) with 358 and 206 turns on the primary and secondary side, respectively. We consider no-load operation. The core is assumed non-linear (Brauer analytical law is used, $b h$ curve depicted in Fig. 2) and isotropic with conductivity $\sigma_{\text {core }}=3.7210^{3} \mathrm{~S} / \mathrm{m}$. The geometry is meshed with 2406 triangles with a classical FE approach. The discretisation is kept constant for all considered 

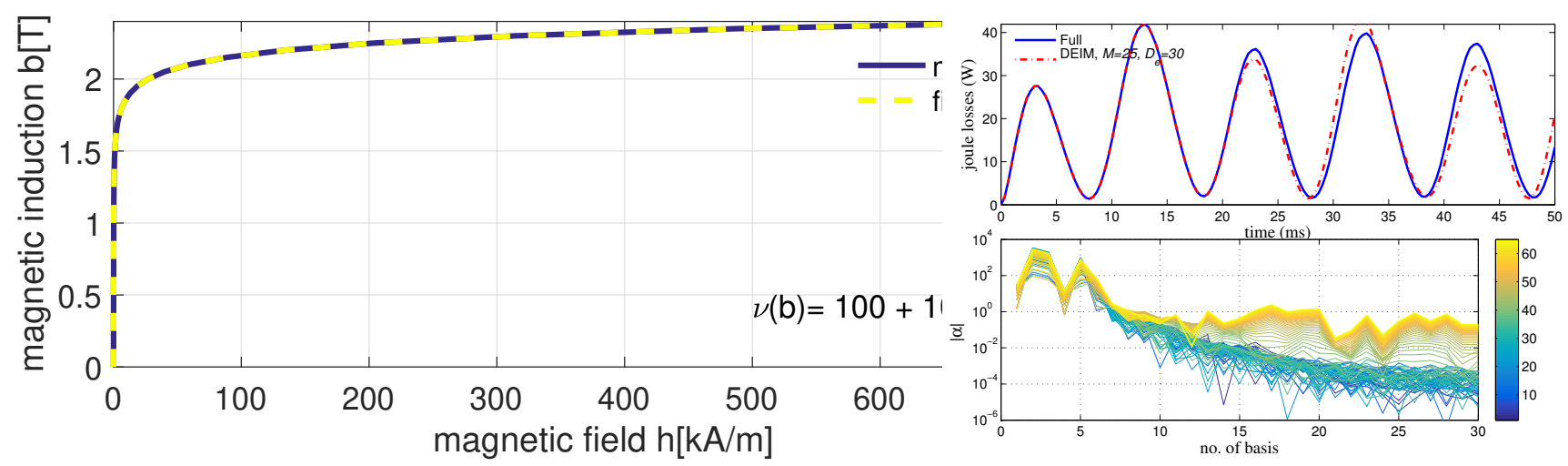

Fig. 2. bh-curve of the core material.

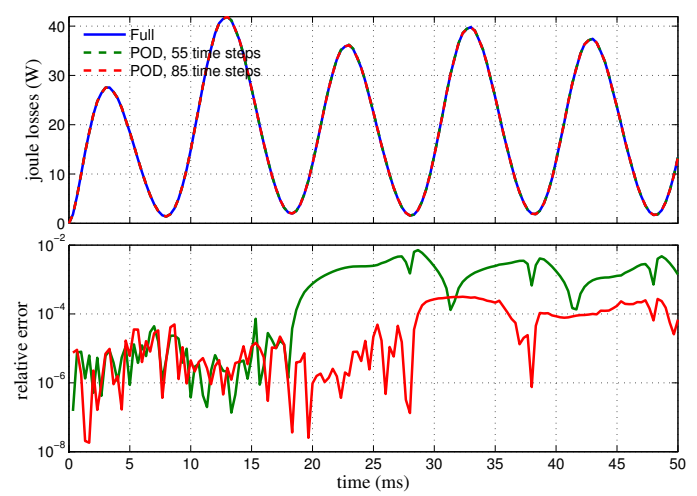

Fig. 3. Joule losses (up) and relative error (down) for the POD with $M=25$, obtained from 55 and 85 time steps.

cases. The primary coil is fed with a $f=50 \mathrm{~Hz}$ sinusoidal current of amplitude $5 \mathrm{~A}$. The problem is time-stepped for 2.5 periods with 60 steps per period, i.e 150 steps in total. The number of unknowns is 1185 .

\section{A. RO modelling with POD}

In POD approach the projection operator $\Psi$ is generated from a snapshot matrix that includes solution vectors $x_{k}$. We have observed from Fig. 3, the time evolution of the joule losses of the power transformer core that, at least first 55 time steps (TS) (18 ms, less than a period) with truncated basis $M=25$ ( $M$ number of first singular value modes greater than a prescribed error tolerance $\epsilon=10^{-6}$ ) are required to approximate the full system accurately. The $L_{2}$ relative error is $0.13 \%$. The accuracy further increases by adding more time solutions $x_{k}$ (i.e. 85 TS corresponds to the $28 \mathrm{~ms}$ ) to the snapshot matrix see Fig 3. The $L_{2}$ relative error becomes $0.01 \%$. Although the POD approach is very accurate, it is as computationally expensive as full system due to the nonlinear iterations (even more if we are not doing repetitive computations as the generation of RO system is expensive).

\section{B. RO modelling with DEIM}

The computational complexity of the non-linear term mentioned in section III, can be greatly reduced by the DEIM
Fig. 4. Case-1 : Joule losses (up) and error indicator $\alpha$ per time step (down) for the DEIM with 55 TS (18 ms) snapshots. Colormap scale (TS): dark blue (initial TS) to dark yellow (final TS).

technique. However, including the eddy currents, the RO models can encounter instability issues. We have considered 5 different cases listed in Table I to observe this. As we already have seen in the previous section that, 55 TS with $M=25$ is enough to represent the full solution, therefore, we have taken this as a starting point of our DEIM-POD simulations. We keep the POD basis number $M=25$ same and change the non-linear basis number $D_{e}$ for all DEIM-POD models by considering two set of snapshots (55 and 85 TS). In Fig 4 and 5 it can be seen that the RO models are inaccurate inspite of having enough (expected) information for the full system approximation. The second figure of Fig 4 and 5 represents the evolution of the coefficients of $\alpha$ for each time step (one curve corresponds to one time step). The oscillation of the interpolation coefficient $\alpha$ values in the same figures also shows that the RO models are indeed unstable, as the evolution of the $\alpha$ values is not decreasing with respect to the index of basis vector. From Fig 6, we can observe that by increasing the snapshot number to 85 TS the error might get reduced but still high, i.e., $1.57 \% L_{2}$ relative error. In order to reduce this error furthermore, we increase the non-linear basis number $D_{e}$ from 25 to 30, keeping same number of snapshots (85 TS) and we observe that the RO model becomes completely false, see Fig 7.

\section{C. $R O$ modelling with $G-P O D$}

In order to cope up with the DEIM-POD instability issue, a Gappy POD based stabilization procedure is implemented with a stabilization indicator $\alpha$. The $\alpha$ values depicted in Fig 8 and 9 for both case- 1 and case- 2 show that, the RO models start becoming unstable after 60 time steps. Therefore, by observing $\alpha$ for several time steps starting from 60 , it can be seen that the minimum value appears at the 24th number of non-linear basis / interpolating function. This indicates the appropriate non-linear basis points selection in order to get the stable RO models. Therefore, by selecting the basis at $D_{e}=24$ in GPOD method the instability problem of the DEIM method can be solved, see Fig 12 and 13. Similarly, for 85 snapshots cases, i.e., case-3, 4 and 5, by observing the unstable time steps $\alpha$ we can choose the non-linear basis number $D_{e}=22,24,29$ 

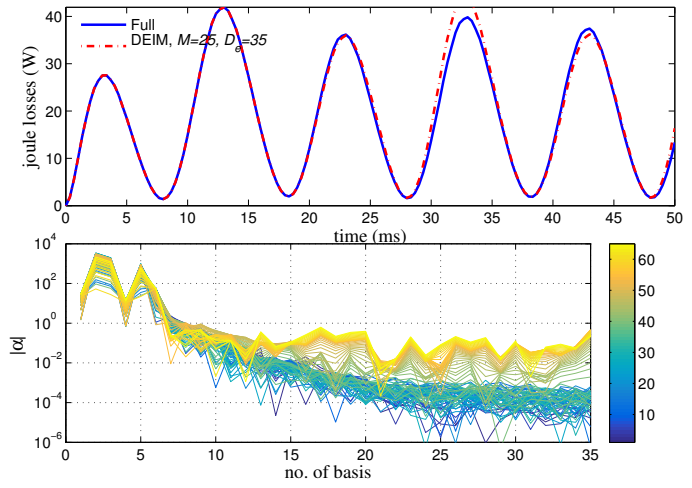

Fig. 5. Case-2 : Joule losses (up) and error indicator $\alpha$ per time step (down) for the DEIM with 55 TS $(18 \mathrm{~ms})$ snapshots. Colormap scale (TS): dark blue (initial TS) to dark yellow (final TS).
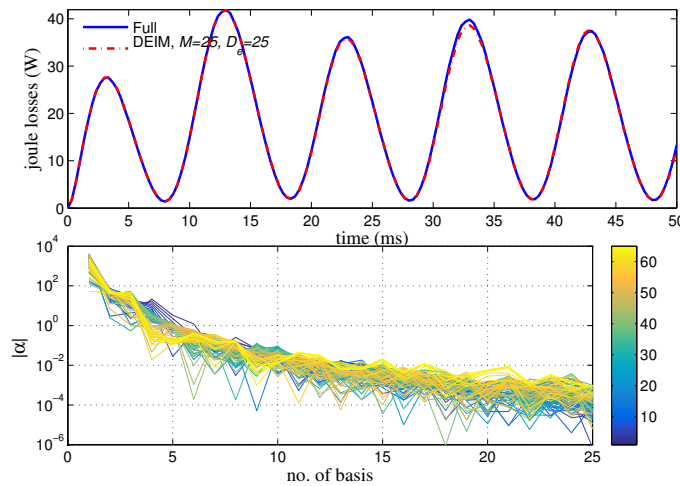

Fig. 6. Case-3 : Joule losses (up) and error indicator $\alpha$ (down) for the DEIM with $85 \mathrm{TS}(28 \mathrm{~ms})$ snapshots. Colormap scale (TS): dark blue (initial TS) to dark yellow (final TS).
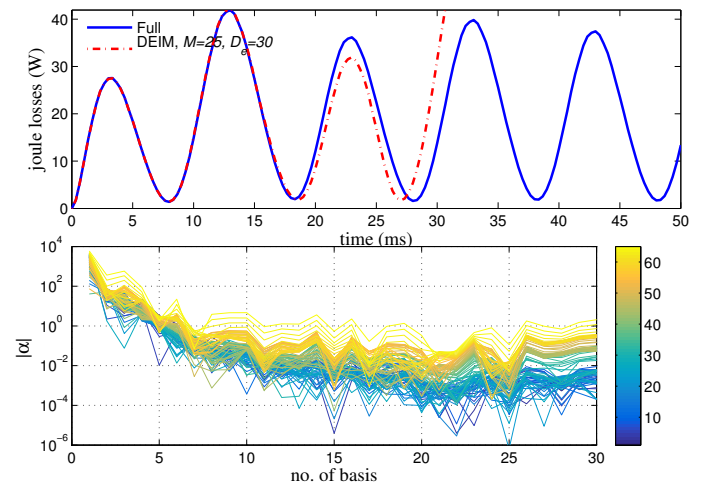

Fig. 7. Case-4 : Joule losses (up) and error indicator $\alpha$ (down) for the DEIM with 85 TS ( $28 \mathrm{~ms})$ snapshots. Colormap scale (TS): dark blue (initial TS) to dark yellow (final TS).
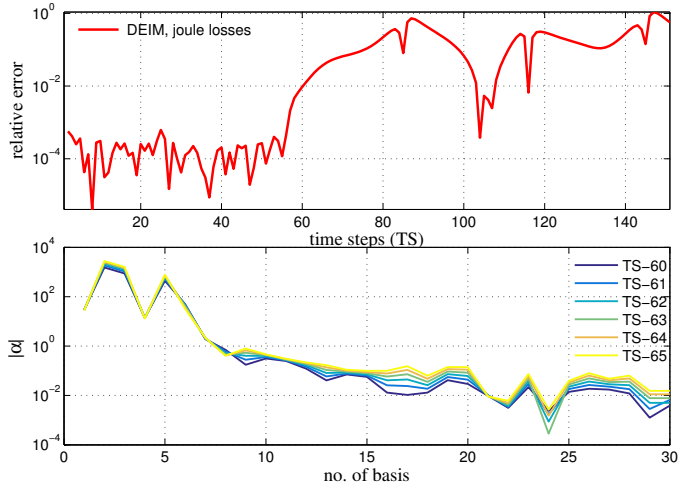

Fig. 8. Case-1 : relative error (top) and error indicator $\alpha$ (bottom) for the DEIM with 55 TS (18 ms) snapshots (analyzed from Fig.4).
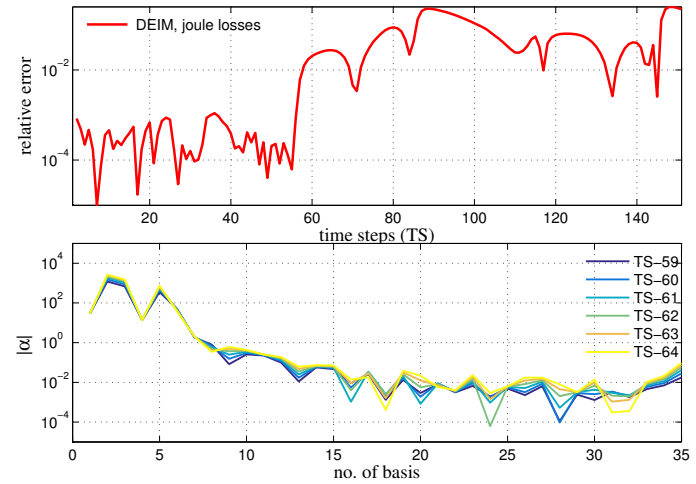

Fig. 9. Case-2 : relative error (top) and error indicator $\alpha$ (bottom) for the DEIM with 55 TS $(18 \mathrm{~ms}$ ) snapshots (analyzed from Fig.5).

(see Fig. 10, 11, ??) respectively in order to stabilize the RO models (see Fig. 14, 15, ??). The $L_{2}$ errors of the joule losses for all the cases are listed in the Table I. The average number of non-linear iterations to converge for full and RO models is 14.

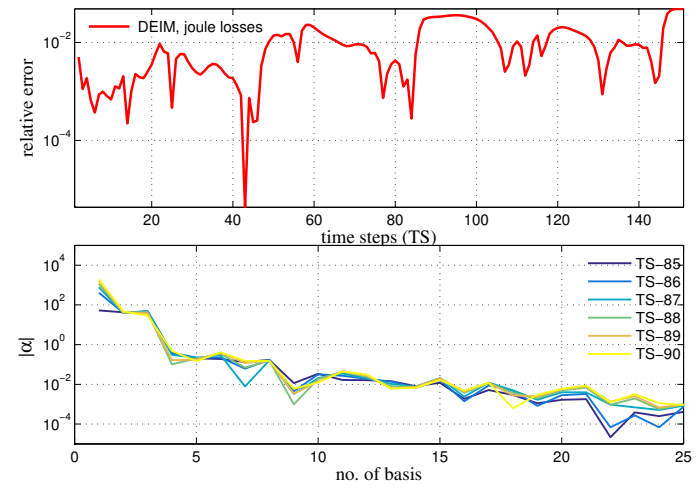

Fig. 10. Case-3 : relative error (top) and error indicator $\alpha$ (bottom) for the DEIM with 85 TS (28 ms) snapshots (analyzed from Fig.6). 

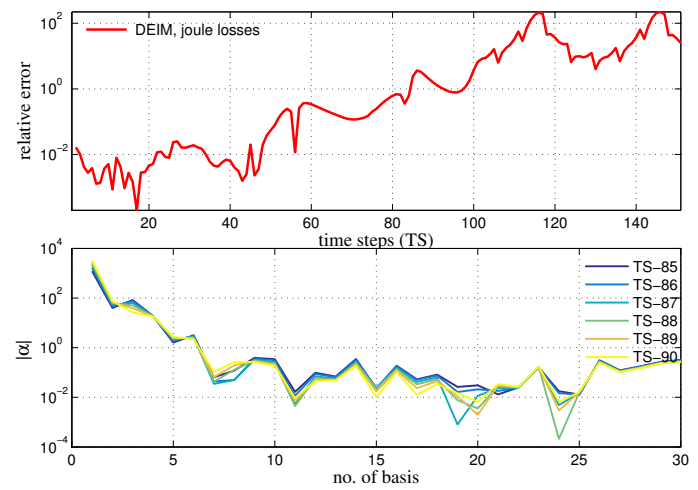

Fig. 11. Case-4 : relative error (top) and error indicator $\alpha$ (bottom) for the DEIM with 85 TS (28 ms) snapshots (analyzed from Fig.7).
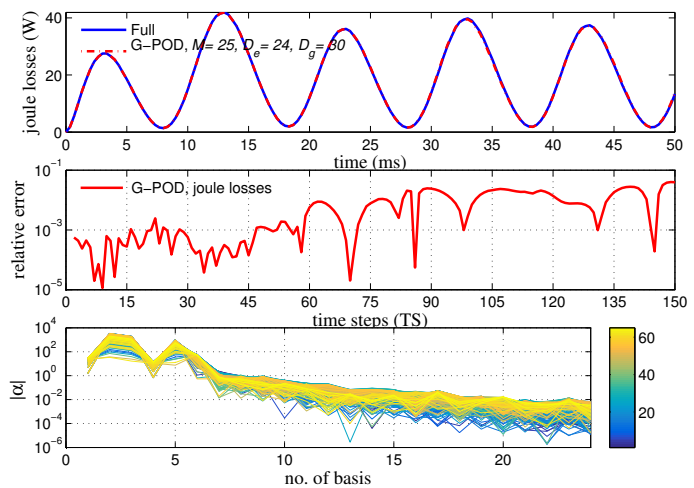

Fig. 12. Case-1 : Joule losses (top), relative error (middle) and error indicator $\alpha$ (bottom) for the G-POD with 55 TS $(18 \mathrm{~ms})$ snapshots. Colormap scale (TS): dark blue (initial TS) to dark yellow (final TS).
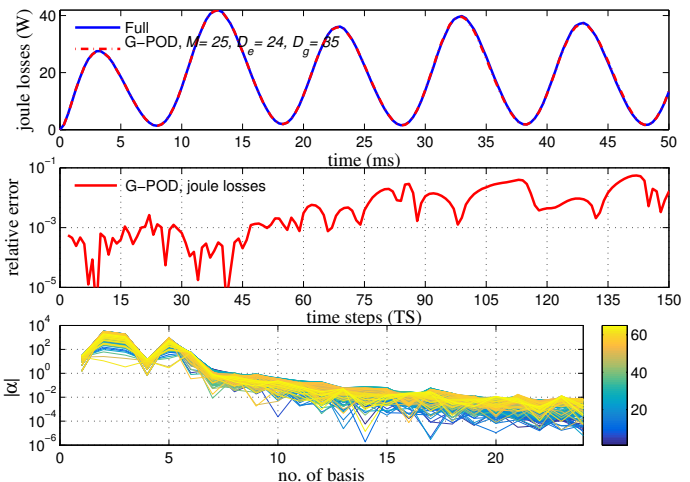

Fig. 13. Case-2 : Joule losses (top), relative error (middle) and error indicator $\alpha$ (bottom) for the G-POD with 55 TS $(18 \mathrm{~ms})$ snapshots. Colormap scale (TS): dark blue (initial TS) to dark yellow (final TS).

TABLE I

$L_{2}$-RELATIVE ERRORS OF RO MODELS ON POWER TRANSFORMER CORE JOULE LOSSES.

\begin{tabular}{|l|l|c|c|c|c|c|}
\hline \multirow{2}{*}{$\begin{array}{l}\text { Case } \\
\text { No. }\end{array}$} & \multirow{2}{*}{ Snapshots } & \multicolumn{2}{|c|}{ DEIM } & \multicolumn{4}{c|}{ GPOD } \\
\cline { 3 - 7 } & & $D_{e}$ & $L_{2}$-error $\%$ & $D_{e}$ & $D_{g}$ & $L_{2}$-error \% \\
\hline 1 & 55 & 30 & 11.69 & 24 & 30 & 0.92 \\
\hline 2 & 55 & 35 & 6.50 & 24 & 35 & 0.95 \\
\hline 3 & 85 & 25 & 1.57 & 22 & 25 & 0.38 \\
\hline 4 & 85 & 30 & diverge & 24 & 30 & 0.44 \\
\hline
\end{tabular}
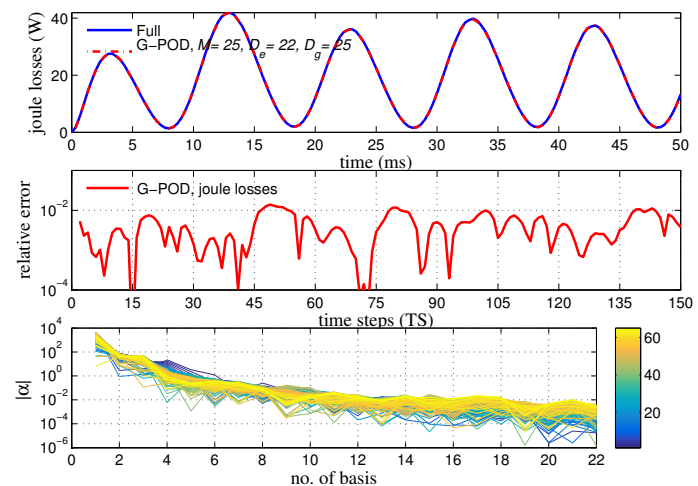

Fig. 14. Case-3 : Joule losses (top), relative error (middle) and error indicator $\alpha$ (bottom) for the G-POD with 85 TS $(28 \mathrm{~ms})$ snapshots. Colormap scale (TS): dark blue (initial TS) to dark yellow (final TS).
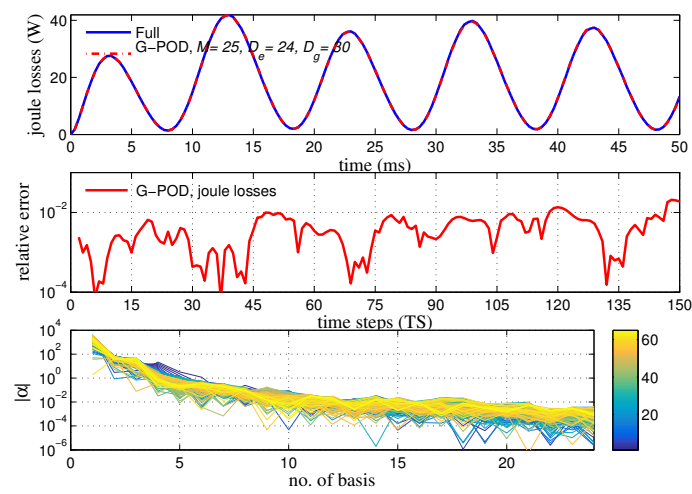

Fig. 15. Case-4: Joule losses (top), relative error (middle) and error indicator $\alpha$ (bottom) for the G-POD with 85 TS $(28 \mathrm{~ms})$ snapshots. Colormap scale (TS): dark blue (initial TS) to dark yellow (final TS).

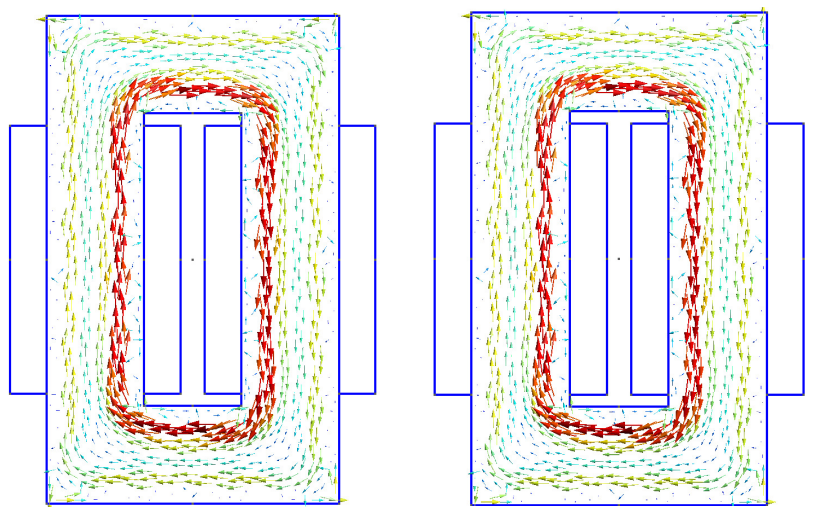

Fig. 16. G-POD with 85 TS $(28 \mathrm{~ms})$ snapshots: Magnetic flux density $b$ for case-4 (left) and case-5 (right) at 95 time steps. 

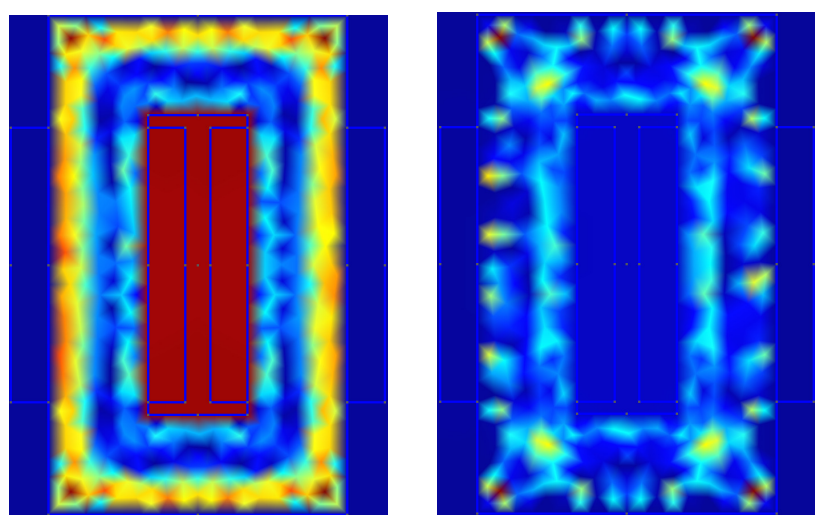

Fig. 17. G-POD with 85 TS $(28 \mathrm{~ms})$ snapshots: Absolute error of $a z$ for case4 (left) and case-5 (right) at 95 time steps. Scale range: $10^{-25}$ (dark blue), $10^{-5}$ (yellow) and $10^{-3}$ (dark red).

\section{3D Three Phase Transformer}

A 3D three phase transformer at no load is studied [?]. The aim is to study the waveform of primary currents versus the time when a sinusoidal voltage supply is imposed. The supply frequency is $50 \mathrm{~Hz}$. Due to the symmetry, only one quarter of the transformer is modeled (fig. 18). The nonlinear behaviour of the magnetic core is considered. The 3D spatial mesh is made of 12636 nodes and 66382 tetrahedra.

For this problem, the currents are unknowns when the voltages are imposed. Due to a larger transient state, the time interval is $1 s$ in order to obtain waveforms of current close to their steady states. The number of time steps per period is 30 and then 1500 on the time interval. The RO models are based on the snaphots extracted to the first three periods which correspond to 90 time steps. The POD reduced subspace $\Psi$ used to approximate the vector $x_{k}$ is composed by the 40 first columns of $\mathcal{U}$ (7). For the DEIM-POD model, if we consider the same size for the reduced subpace $W$ of the nonlinear term (13), the solution of the reduced model diverge after 6 periods. Then, the efficiency of the DEIM-POD model can be improved with $D_{e}=70$. Nevertheless, the precision of the solution decreases as function of time. For the G-POD model, we consider $D_{g}=70$ and $D_{e}=40$. In this case, the RO model is efficient on the time interval. The $L_{2}$ relative error of the currents is close to $2 \%$ for 1500 time steps. Figure (19) presents the waveforms of the currents at the beginning and at the end of the time interval obtained from the full and G-POD models.
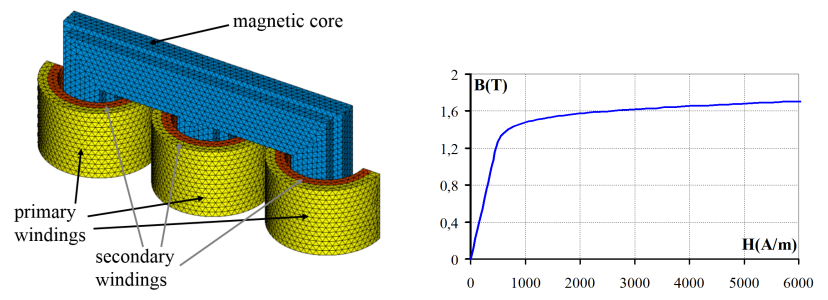

Fig. 18. Mesh (left) and bh curve (right) of the three-phase transformer considered for validation.
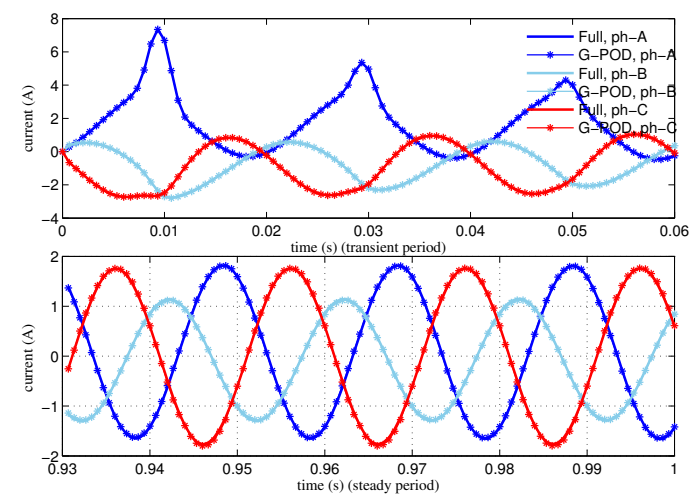

Fig. 19. Current waveform of the Full and G-POD for currents 1A, 2A and 3A. Transient period (up) and steady state period (down).

The distributions of the magnetic flux density obtained from the full model and the error distribution of fields between the full and G-POD models are presented for the time step corresponding to the highest level of saturation in figure 20 . The magnitude of error is small compared to this of the magnetic flux density. The maximal errors are located on the corners of the magnetic core.

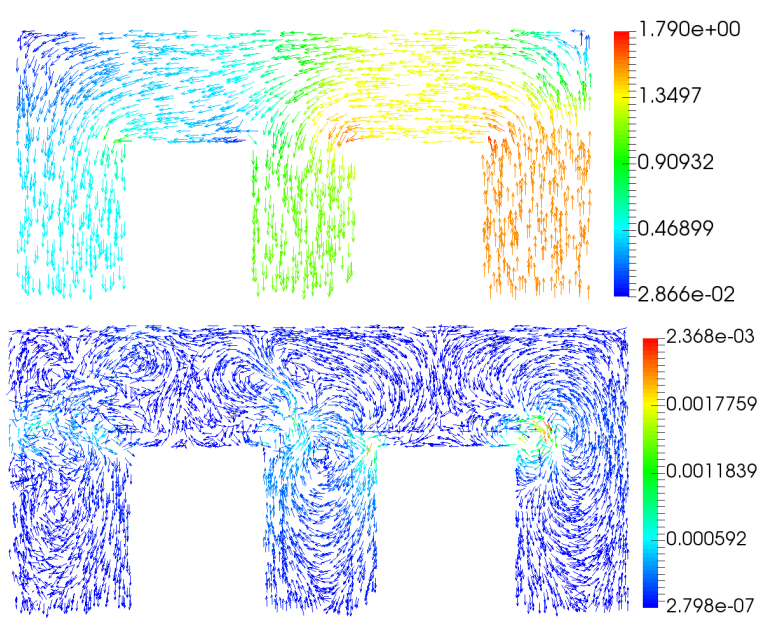

Fig. 20. Distribution of the magnetic flux density (T), full model (up) and error (down).

Figure 21 presents the localisation of the non-linear components selected by the G-POD approach. These components correspond to a set of edges. We can observe that the most edges are located in the zones where the saturation of the magnetic core is high.

Figure 22 presents the field distribution corresponding to $\operatorname{curl} \Psi$ for the first four vector in the magnetic core. The distribution corresponding to curl $\Psi_{1}$ and $\operatorname{curl} \Psi_{2}$ are close to a physical distribution of the magnetic flux density encountered in a transformer. The distributions corresponding to $\operatorname{curl} \Psi_{3}$ and $\operatorname{curl} \Psi_{4}$ have no physical meaning except that they enable us to better take into account the saturation at the corner of the magnetic core.

In terms of computational time, with a time interval width of $1 s$ and 1500 time steps, the full model requires $164 \mathrm{~min}$. The 


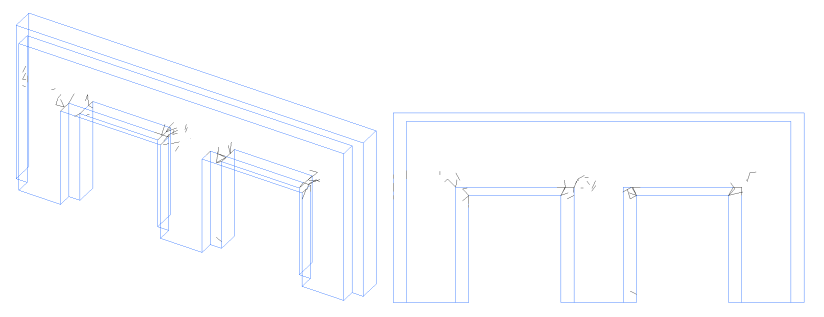

(a) 3D view

(b) $2 \mathrm{D}$ view

Fig. 21. Localisation of the non-linear components

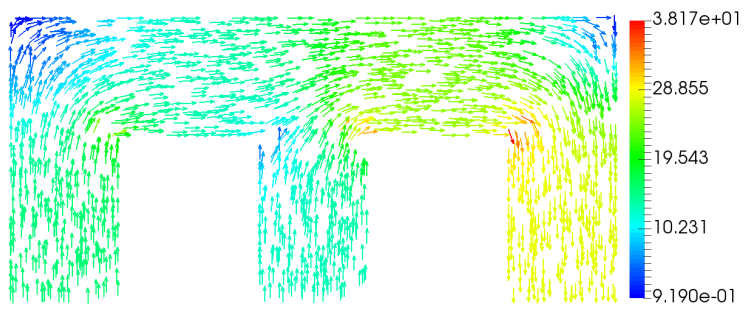

(a) $\operatorname{curl} \Psi_{1}$

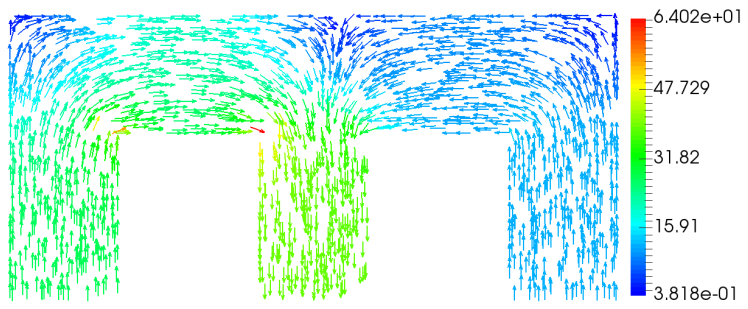

(b) $\operatorname{curl} \Psi_{2}$

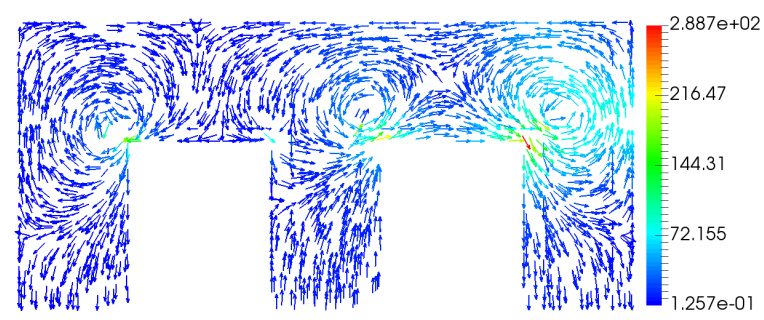

(c) $\operatorname{curl} \Psi_{3}$

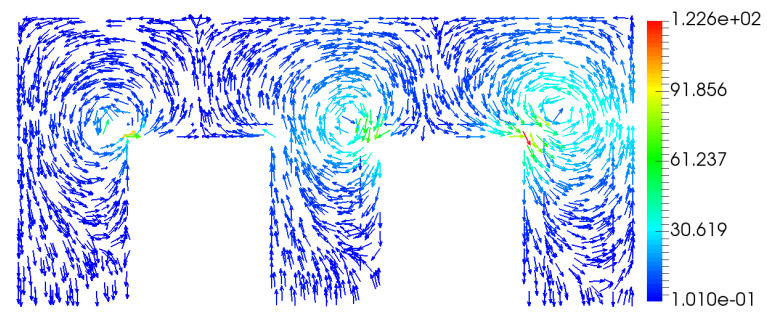

(d) $\operatorname{curl} \Psi_{4}$

Fig. 22. Distribution of the $\operatorname{curl} \Psi$

speed up obtained from the GPOD-POD model is 5 . This speed up is penalized due to the projection of the reduced solution on the FE basis in order to build the distribution of fields at each time step. In the case where the distribution of fields are not required, the speed up is 150 .

\section{CONCLUSION}

In this paper, we have proposed three RO approaches: POD, DEIM and GPOD to model a non-linear eddy current problem.
A GPOD based stabilization technique has been proposed with an error indicator to improve the stability problem of the DEIM method. It has been observed that GPOD can fix the unstable RO models with considerable accuracy, i.e. relative error less than $0.5 \%$. Although the POD technique is much more accurate than the DEIM and GPOD method, due to the computational complexity the gain of this technique for generating RO models is less. The DEIM has been proven to be highly computationally efficient in case of a non-linear problem. However, GPOD can greatly deal with the system instability when the system incorporate with the eddy current problem. The GPOD method is as computational efficient as the DEIM method, less accurate but stable.

\section{REFERENCES}

[1] Hasan, MD R., Sabariego, R. V., Geuzaine, C., Paquay, Y.: Proper orthogonal decomposition versus Krylov subspace methods in reducedorder energy-converter models. In Proc. of IEEE Inter. Ener. Conf., pp. $1-6,(2016)$.

[2] Henneron, T., Clénet, S.: Model order reduction of quasi-static problems based on POD and PGD approaches. The European Physical JournalApplied Physics, vol. 64, no. 2 (2013).

[3] Henneron, T., Clénet, S.: Model order reduction of electromagnetic field problem coupled with electric circuit based on proper orthogonal decomposition. In Proc. OIPE, Ghent, Belgium, (2012).

[4] Schmidthausler, D., Clemens, M.: Low-order electroquasistatic field simulations based on proper orthogonal decomposition. IEEE Trans. Mag. vol. 48, no. 2, pp. 567-570, (2012).

[5] Banerjee, S., Cole, J. V., Jensen, K. F. : Nonlinear Model Reduction Strategies for Rapid Thermal Processing Systems. Trans. Semicond. Manufac., vol. 11, pp. 266-275, (1998).

[6] Chen, Y.: Model Order Reduction for Nonlinear Systems. Master's thesis, MIT, (1999).

[7] White, J. K.: A Trajectory Piecewise-Linear Approach to Model Order Reduction of Nonlinear Dynamical Systems. Doctoral dissertation, MIT, (2003).

[8] Albunni, M.N., Rischmuller, V., Fritzsche, T., Lohmann, B.: Model-order reduction of moving nonlinear electromagnetic devices. IEEE Trans. Mag., vol. 44, no. 7, pp. 1822-1829, (2008).

[9] Barrault, M., Maday, Y., Nguyen, N.C. and Patera, A.T.: An empirical interpolation method: Application to efficient reduced-basis discretization of partial differential equations. C. R. Acad. Sci. Paris, vol. 339, no. 9, 2004, pp. 667-672, (2004).

[10] Chaturantabut, S., Sorensen, D. C: Nonlinear model reduction via discrete empirical interpolation. SIAM J. Sci. Comp., vol. 32, no. 5, pp. 2737-2764, (2010).

[11] Henneron, T., Clénet, S.: Model order reduction of non-linear magnetostatic problems based on POD and DEI methods. IEEE Trans. Mag., vol. 50, no. 2, pp. 33-36, (2014).

[12] Peherstorfer, B., Butnaru, D., Willcox, K., Bungartz, H. J.: Localized discrete empirical interpolation method. SIAM J. Sci. Comp., vol. 36, no. 1, pp. A168-A192, (2014).

[13] Montier, L., Henneron, T., Clénet, S., Goursaud, B.: Robust model order reduction of an electrical machine at startup through reduction error estimation. Int. J. Numer. Model., DOI: 10.1002/jnm.2277, (2017).

[14] Everson, R., Sirovich, L.: KarhunenLoeve procedure for gappy data. JOSA A, vol. 12, no. 8, pp. 1657-1664, (1995).

[15] Willcox, K.: Unsteady flow sensing and estimation via the gappy proper orthogonal decomposition. Computers and fluids, vol. 35, no. 2, pp. 208226, (2006).

[16] Yildirim, B., Chryssostomidis, C., Karniadakis, G. E.: Efficient sensor placement for ocean measurements using low-dimensional concepts. Ocean Modelling, vol. 27, no. 3, pp. 160-173, (2009).

[17] Sato, Y., Igarashi, H.: Model reduction of three-dimensional eddy current problems based on the method of snapshots. IEEE Trans. Magn., vol. 49, no. 5, pp. 1697-1700, (2013). 